\title{
Long-term Results of the Thrust Plate Prosthesis, 21-year Follow-up: A Case Report and Literature Review
}

\author{
Hany Elbardesy ${ }^{1}$, Rehan Gul ${ }^{1}$
}

\section{Learning Point of the Article:}

The TPP is not an alternative to THA. It may be indicated in some cases for young patients where, several revision surgeries are expected, a loosened TPP can be revised later with a stemmed implant into an unaltered diaphysis and only slightly changed metaphyseal bone.

\section{Abstract}

Introduction: The thrust plate prosthesis (TPP) is a type of cementless hip replacement. Aimed to preserve femoral diaphyseal bone, it was favored by some orthopedic surgeons in younger patients as they could potentially undergo multiple revision arthroplasties during their lifetime. Of particular note, the preserved diaphyseal bone allows for the implantation of a subsequent primary total hip arthroplasty (THA).

Case Report: We reported on a 64-year-old male patient who underwent thrust plate prosthesis (TPP) implantation 21 years ago (1999) for the treatment of primary osteoarthritis $(\mathrm{OA})$ of the right hip joint. At 21 -year follow-up, he had not developed any post-operative complications, and he reported a SF12 score of 32 and Oxford hip score of $47 / 48$.

Conclusion: TPP proved to be successful clinically and radiologically, especially in the young patient. It loads directly to the cortex of the proximal femoral metaphysis as in a native hip joint. It is a bone preserving prosthesis which allows for good bone stock in the case of revision surgery that was a victim of commercial considerations.

Keywords: Thrust plate prosthesis, thrust plate prosthesis, arthroplasty.

\section{Introduction}

The thrust plate prosthesis (TPP) was a hip implant that allowed for the preservation of the bone of the femoral neck and as such was used in younger patients $[1,2]$. The TPP was fitted to the metaphyseal part of the proximal femur after removal of a small amount of bone stock and results in near physiological load transfer and strain distribution and allows for satisfactory biomechanical hip reconstruction $[3,4,5]$. While patients with short-stem hip implants can return to a good level of activity postoperatively, and in some cases return to pre-operative levels of participation, femoral short stems can result in a higher incidence of malalignment and subsidence $[6,7]$. Although the TPP is not available anymore for commercial reasons, its legacy as a successful prosthesis lives on, supported by literature reporting favorable outcomes at long-term follow-up $[5,8,9$, $10,11]$. TPP was proved to be clinically and radiologically successful. However, because of the complexity of the prosthesis, with several parameters having to be addressed during surgery, the technique for insertion has a steep learning curve [12].

\section{Case Report}

We report the case of a 64-year-old male patient who underwent TPP implantation 21 years ago (1999) for treatment primary osteoarthritis (OA) of the right hip joint. At 21-year follow-up, the patient had not developed any complications and reported a SF12 score of 32 and an Oxford hip score of 47/48. TPP has shown to be an excellent therapeutic option and alternative to standard stem total hip replacement (THR) for young patients with symptomatic hip OA as it restores the biomechanical and physiological load of the native hip. His current plain radiograph (Fig. 1) and CT scan (Fig. 2) are provided.

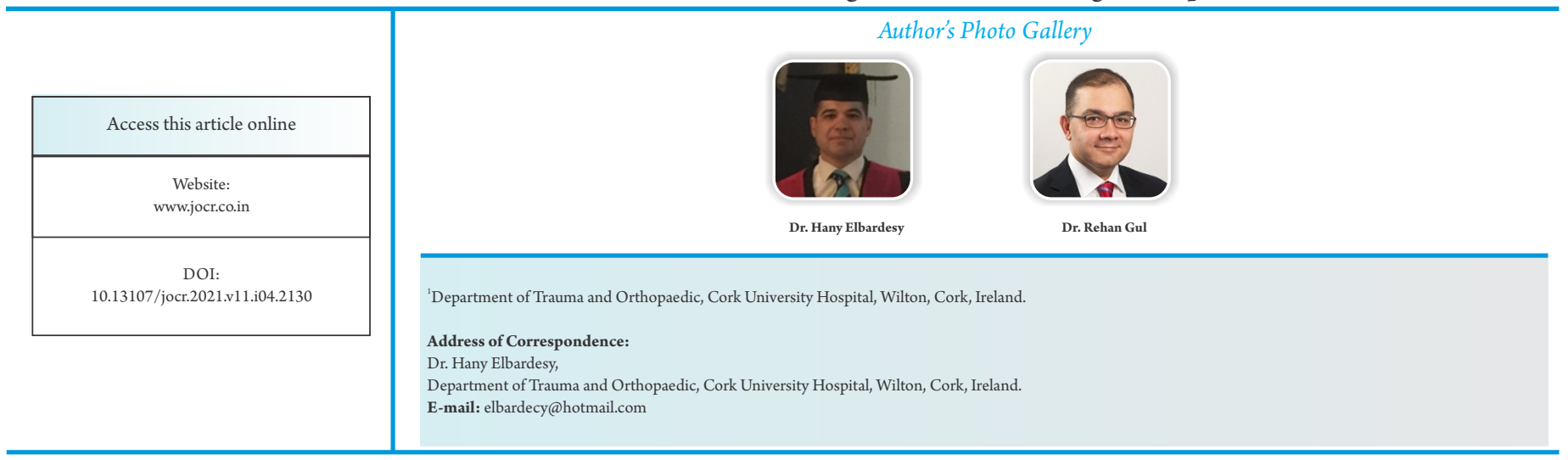

Journal of Orthopaedic Case Reports | pISSN 2250-0685 | eISSN 2321-3817 | Available on www.jocr.co.in | doi:10.13107/jocr.2021.v11.i04.2130 This is an Open Access article distributed under the terms of the Creative Commons Attribution Non-Commercial License (http://creativecommons.org/licenses/by-nc/3.0) which permits unrestricted non-commercial use, distribution, and reproduction in any medium, provided the original work is properly cited. 


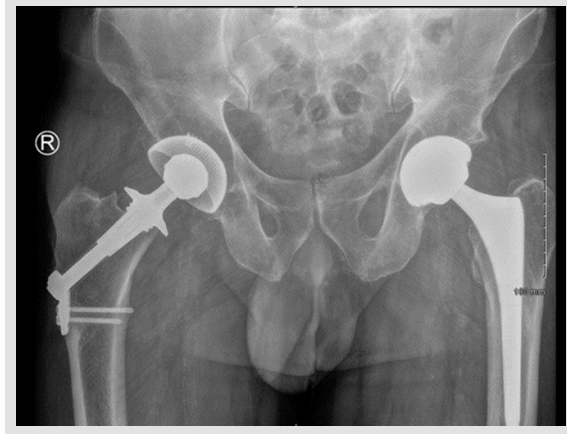

Figure 1: Anteroposterior pelvis radiograph shows the triphenylphosphine in situ.

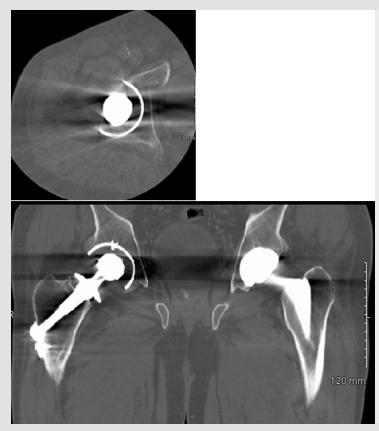

Figure 2: Computed tomography scan of the right hip

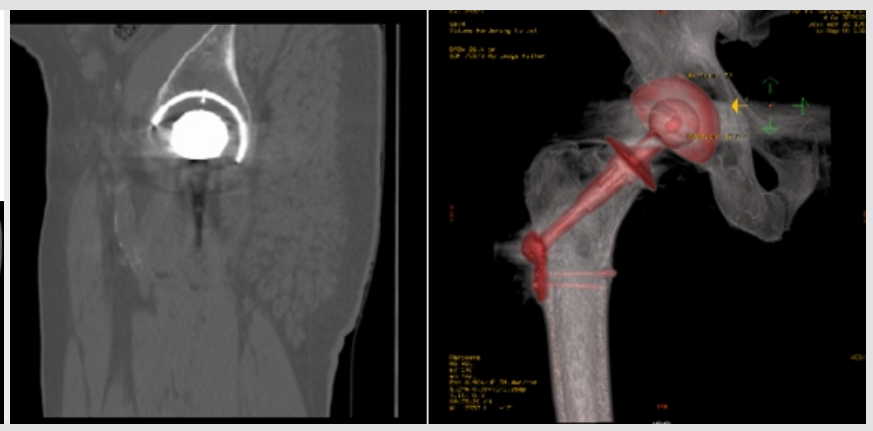

follow-up, which is considered favorable and comparable to cementless or cemented THA [7]. TPP has also demonstrated a dislocation rate of $3.3 \%$ which is comparable to the standard THA (0.3-2.55\%) [24]. This may be due to the TPP position on a shaved neck of femur, which may lead to mechanical impingement. In addition, the position of the TPP on the femoral neck does not permit any alteration of either the femoral shaft-neck angle or the anteversion angle [23]. Certain studies do not support the mechanical impingement hypothesis due to the non-constrained rotational movement of the hip with a TPP. Bone resorption at the inner side of the thrust plate was previously explained as an adjustment of the bone to the biomechanical changes [22]. It was not a sign of loosening, however, it could be justified by stress shielding [23]. This would disprove the biomechanical theories of Jacobs and Huggler and highlight the argued biomechanical principles of the TPP $[3,15]$. Revision rates and aseptic loosening were both higher in TPPs than with primary THA. An evaluation of the Norwegian prosthesis register showed that young age is a risk factor for aseptic loosening [25]. This higher rate of loosening is related to higher levels of activity in young patients with subsequent greater mechanical load on the prosthesis $[21,26]$. Cementless THA has shown superior survival rates compared to TPP in younger patients $[27,28]$. Fink et al. could not find any significant survival rate difference between the two age groups (older or younger than 50 years old), which goes against the hypothesis that young age is a risk factor for early prosthesis failures [23]. Some authors reviewing the necrosis of the femoral head suggested that the etiological factors of femoral head necrosis could lead to an early loosening of the hip arthroplasty by diminishing the biomechanical and/or biological quality of hip bone $[10,18]$. These theories could help explain the high level of aseptic loosening associated with the TPPs if the loosening rates in patients with femoral head necrosis and rheumatoid arthritis were higher than in those with diagnosed dysplastic hips and hip osteoarthritis. Despite this, the total revision rate of the four cohorts did not show any significant difference. Moreover, the mechanical failure rate of TPP in rheumatoid arthritis and avascular necrosis patients was patients reported an average HHS of 91.2 points after 5 years 
$6.8 \%$ and $0 \%$, respectively, which was not significantly higher than those patients with hip osteoarthritis (3.8\%) and with hip dysplasia (6.7\%) [23]. Therefore, it could be assumed that the reason for the higher loosening rates could found in the structure and the principles of fixation of the TPP itself. Lateral thigh pain is another issue with the TPP after implantation and has been reported in up to $15 \%$ of patients in some studies [29]. Despite the many advantages to TPP implantation, the paucity of long-term survivorship data raises concern. Our case report provides one of the longest reported follow-ups of a TPP patient, and it would be premature to draw a meaningful conclusion based solely on this report.

\section{Conclusion}

TPP was proved to be clinically and radiologically successfully, especially in the young patient. This may be due in part to it loading directly onto the cortex of the proximal femoral metaphysis as in a native hip joint, and due to its preservation of proximal femoral bone stock, allowing for primary THA in the future. The TPP became a victim to commercial and marketing consideration and despite its favorable mid-term outcomes, its implantation requires meticulous technique and experienced hands.

\section{Clinical Message}

The TPP is not an alternative to THA. It may be indicated in some cases for young patients where, several revision surgeries are expected, a loosened TPP can be revised later with a stemmed implant into an unaltered diaphysis and only slightly changed metaphyseal bone.

\section{References}

1. Buergi ML, Stoffel KK, Jacob HA, Bereiter HH. Radiological findings and clinical results of 102 thrust-plate femoral hip prostheses: A follow-up of 2 to 8 years. J Arthroplasty 2005;20:108-17.

2. Söderman P, Malchau H, Herberts P. Outcome after total hip arthroplasty: Part I. General health evaluation in relation to definition of failure in the Swedish National Total Hip Arthoplasty register. Acta Orthop Scand 2000;71:354-9.

3. Huggler AH, Jacob HA, Bereiter H, Haferkorn M, Ryf C, Schenk R. Long-term results with the uncemented thrust plate prosthesis (TPP). Acta Orthop Belg 1993;59:21523.

4. Karatosun V, Unver B, Gunal I. Hip arthroplasty with the thrust plate prosthesis in patients of 65 years of age or older: 67 patients followed 2-7 years. Arch Orthop Trauma Surg 2008;128:377-81.

5. Karatosun V, Unver B, Gültekin A, Günal I. Thrust plate prosthesis for proximal femoral deformity: A series of 15 patients. Acta Orthop Traumatol Turc 2010;44:437-42.

6. Schmidutz F, Grote S, Pietschmann M, Weber P, Mazoochian F, Fottner A, et al. Sports activity after short-stem hip arthroplasty. Am J Sports Med 2012;40:425-32.

7. Shin YS, Suh DH, Park JH, Kim JL, Han SB. Comparison of specific femoral short stems and conventional-length stems in primary cementless total hip arthroplasty. Orthopedics 2016;39:e311-7.

8. Ishaque BA, Wienbeck S, Stürz H. Midterm results and revisions of the thrust plate prosthesis (TPP). Z Orthop Ihre Grenzgeb 2004;142:25-32.
9. Diederix LW, Van Winterswijk PJ, Schouten SB, Bakx PA, Huij J. The thrust plate prosthesis: Long-term clinical and radiological results. Acta Orthop Belg 2013;79:293-300.

10. Corner JA, Rawoot A, Parmar HV. The thrust plate prosthesis in the treatment of osteoarthritis of the hip. Clinical and radiological outcome with minimum 5-year follow-up. Hip Int 2008; 18:88-94.

11. Yasunaga Y, Goto T, Hisatome T, Tanaka R, Yamasaki T, Ochi $\mathrm{M}$. Bone-preserving prosthesis with a single axis for treating osteonecrosis of the femoral head: Midterm results for the thrust plate hip prosthesis. J Orthop Sci 2003;8:818-22.

12. Kaegi M, Buergi ML, Jacob HA, Bereiter HH. The thrust plate hip prosthesis: A follow-up of 15-20 years With 102 implants. J Arthroplasty 2016;31:1035-9.

13. Brittain R, Young E, Mccormack V, Swanson M. 16th Annual Report 2019: National Joint Registry for England, Wales, Northern Ireland and the Isle of Man," No. December 2018; 2019.

14. Fottner A, Schmid M, Birkenmaier C, Mazoochian F, Plitz W, Volkmar J. Biomechanical evaluation of two types of short-stemmed hip prostheses compared to the trust plate prosthesis by three-dimensional measurement of micromotions. Clin Biomech 2009;24:429-34.

15. Jacob HA, Huggler AH. An investigation into biomechanical causes of prosthesis stem loosening within the proximal end of the human femur. J Biomech 1980;13:159-73.

16. Christiansen JD, Laursen MB, Ejaz A, Nielsen PT. Bone 
remodelling of the proximal femur after total hip arthroplasty with 2 different hip implant designs: 15 years follow-up of the thrust plate prosthesis and the Bi-Metric stem. HIP Int 2018;28:606-12.

17. Zelle BA, Gerich TG, Bastian L, Shuler FD, Pape HC, Krettek C. Total hip arthroplasty in young patients using the thrust plate prosthesis: Clinical and radiological results. Arch Orthop Trauma Surg 2004;124:310-6.

18. Fink B, Schneider T, Conrad S, Jaeger M, Protzen M, Rüther W. The thrust plate prosthesis in patients with aseptic osteonecrosis of the femoral head. Arch Orthop Trauma Surg 2002;122:499-505.

19. Bereiter H, Bürgi M, Schenk R. Finite element investigations of the proximal femur after implantation of the thrust plate prosthesis compared with findings in a post-mortem histological specimen and in radiological follow-Up examinations. In: The Thrust Plate Hip Prosthesis. Berlin, Heidelberg: Springer Berlin Heidelberg; 1997.p.48-62.

20. Schenk RK, Hauser R, Huggler AH, Jacob HA. Histology of the thrust plate bone interface. In: The Thrust Plate Hip Prosthesis. Berlin, Heidelberg: Springer Berlin Heidelberg; 1997. p. 63-73.

21. Fink B, Siegmüller C, Schneider T, Conrad S, Schmielau G, Rüther W. Short- and medium-term results of the thrust plate prosthesis in patients with polyarthritis. Arch Orthop Trauma Surg 2000;120:294-8.

22. Menge M. Bone remodelling of the proximal femur after implantation of a thrust plate prosthesis. In: The Thrust
Plate Hip Prosthesis. Berlin, Heidelberg: Springer Berlin Heidelberg; 1997.p.98-106.

23. Fink B, Wessel S, Deuretzbacher G, Protzen M, Ruther W. Midterm results of "thrust plate" prosthesis. J Arthroplasty 2007;22:703-10.

24. Prietzel T, Hammer N, Schleifenbaum S, Adler D, Pretzsch M, Köhler L, et al. Der Einfluss der Kapselrekonstruktion auf die Luxationsrate nach primärer Hüftendoprothetik: Eine retrospektive Analyse von 1972 Fällen. Z Orthop Unfall 2014;152:130-43.

25. The Norwegian Hip Fracture Register, Annual Report; 2010. Available from: http://www.nrlweb.ihelse.net/eng. [Last accessed on 2010 Jun ???].

26. Miki H, Sugano N, Yonenobu K, Tsuda K, Hattori M, Suzuki N. Detecting cause of dislocation after total hip arthroplasty by patient-specific four-dimensional motion analysis. Clin Biomech 2013;28:182-6.

27. Australian Orthopaedic Association National Joint Replacement Registry. Australian Orthopaedic Association National Joint Replacement Registry (AOANJRR). Hip Knee Shoulder Arthroplasty Annual Report; 2018.p. 1-444.

28. NI, IM. National Joint Registry for England, Wales, 15th Annual Report 2018. Vol. 1821. England: National Joint Registry for England; 2018.p. 218.

29. Steens W, Daele RV, Simon U, Goetze C. Clinical and radiological mid-term results of the thrust plate prosthesis. Int Orthop 2009;33:1519-24.
Conflict of Interest: Nil

Source of Support: Nil

Consent: The authors confirm that informed consent was obtained from the patient for publication of this case report

\section{How to Cite this Article}

Elbardesy H, Rehan Gul R. Long-term Results of the Thrust Plate Prosthesis, 21-year Follow-up: A Case Report and Literature Review. Journal of Orthopaedic Case Reports 2021 April;11(4): 10-13 